\title{
Effet de quatre types de fertilisants sur la croissance et la productivité de deux génotypes de maïs (Zea mays L.) en cas d'un semis tardif à Korhogo au Centre-Nord de la Côte d'Ivoire
}

\author{
Laopé Ambroise Casimir SIENE*, Mohamed DOUMBOUYA, \\ Mohamed Sahabane TRAORE, Mariame CONDE, Tah Valentin Felix N'GUETTIA et \\ Mamadou KONE
}

UFR des Sciences Biologiques / Département de Biologie Végétale, Université Peleforo GON COULIBALY, Korhogo, Côte d'Ivoire (BP 1328 Korhogo / www.univ-pgc.edu.ci)

*Auteur correspondant ; E-mail : s.silamca@gmail.com ; Tel :00225 08823034

\section{RESUME}

Face à un appauvrissement des sols et une irrégularité des pluies du fait du changement climatique, une étude a été entreprise sur la culture du maïs au Centre-Nord de la Côte d'Ivoire. Elle avait pour objectif d'évaluer l'influence de quatre types de fumures sur le potentiel agronomique de deux génotypes de maïs (Zea mays L.) en cas de contrainte hydrique de fin de cycle et d'infertilité des sols. Le dispositif expérimental a été un split-plot, dont le premier facteur a été les fertilisants et le second, la variété. Les résultats ont montré que les fumures ont influencé les durées des phases végétative et reproductive en plus du rendement. Les fumures fiente de poulet et déjection de bœuf ont permis d'obtenir un rendement qui est égal à six fois celui obtenu avec le témoin de la variété à grain noir malgré la contrainte hydrique. Aussi, la fumure fiente de poulet a eu une tendance à réduire le cycle de culture et favoriser une meilleure croissance et un bon développement des plantes. L'utilisation de ces fumures en culture du maïs, pourrait constituer une alternative aux conséquences drastiques du changement climatique.

(C) 2020 International Formulae Group. All rights reserved.

Mots clés : Fertilisants, maïs (Zea mays L.), potentiel agronomique, rendement, contrainte hydrique.

\section{Effect of four types of fertilizers on the growth and productivity of two maize genotypes (Zea mays L.) in the event of late planting in Korhogo in North- Central Côte d'Ivoire}

\begin{abstract}
Faced with soil depletion and irregular rains due to climate change, a study was undertaken on maize cultivation in North-Central Côte d'Ivoire. Its objective was to assess the influence of four types of manure on the agronomic potential of two maize genotypes (Zea mays L.) in cases of water stress and soil infertility. The experimental set-up was a split-plot, the first five-level factor being fertilizers and the second two-level factor being variety. Observations were made on phenology, growth parameters and yield. The results showed that manure influenced vegetative and reproductive time, growth parameters and yield. In terms of yield, chicken droppings and beef droppings were the most effective. They yielded six times the yield obtained with the
\end{abstract}


black-grain variety control despite the water constraint at the end of the cycle. Also, chicken manure has had a tendency to reduce the growing cycle and promote better growth and plant development. The use of these manure in maize cultivation could be an alternative to the drastic consequences of climate change.

(c) 2020 International Formulae Group. All rights reserved.

Keywords: Fertilizers, corn (Zea mays L.), agronomic potential, yield, water constraint.

\section{INTRODUCTION}

En Afrique Subsaharienne, le maïs est produit principalement pour l'alimentation humaine, soit frais ou en produit transformé. En outre, le maïs sert à l'alimentation animale et a des utilisations industrielles sous forme d'amidon, de farine, d'éthanol, de sirop de cuisson et croustillante (Tshiabukole, 2018). En Côte d'Ivoire, sa production est passée de 661285 tonnes en 2013 à 1025000 tonnes en 2017 (FAO, 2017) et cette culture fait ainsi, l'objet d'une spéculation agricole. Cependant, la majorité de la production nationale est réalisée par des petits producteurs agricoles, dont la plupart produit 1 à 2 tonnes par campagne agricole (Boone et al., 2008). C'est une culture facile à produire, mais, très sensible au manque d'eau. Elle nécessite une pluviométrie variant de 800 à $1200 \mathrm{~mm}$ et un ensoleillement important qui réduit le parasitisme (Hoopen et Maïga, 2012). En effet, le concept de changement climatique devenu aujourd'hui une réalité, a pour conséquences une distribution aléatoire des pluies et une incidence plus grande de la sécheresse sur les productions agricoles (Amigues et al., 2006), la vulnérabilité des sols et des cultures (Tabetaoul et Bessaoud, 2009) entrainant la dégradation avancée des terres cultivables (Assié et al., 2008).

Dans les zones de production céréalières comme le Nord de la Côte d'Ivoire, les dates de semis sont régulièrement décalées du fait du retard observé dans le démarrage de la saison des pluies (Akanvou et al., 2006). De plus, les pauses pluviométriques enregistrées au cours de la saison culturale ont différents effets dépressifs sur la croissance, le développement et la productivité des céréales en général et du maïs en particulier (Diatta, 2007). Des travaux ont aussi montré que, chez le maïs le rendement maximal potentiel est réduit en cas d'une contrainte hydrique (Hoopen et Maiga, 2012 ; Tshiabukole, 2018).
Par ailleurs selon Baboy et al. (2015), un semis tardif se traduit par un ralentissement de la croissance. Ce qui se traduit par une perte de potentiel de rendement. Ainsi, face à l'importance que cette culture représente dans la lutte contre l'insécurité alimentaire, il est nécessaire de faire recours à des pratiques culturales permettant de relever le niveau de fertilité des sols et d'utiliser des variétés précoces avec une bonne tolérance à la sécheresse dans les zones à risques. La fumure minérale longtemps utilisée comme une solution, a montré ses limites du fait de son effet polluant et de son coût élevé (Kiye Nkoy-Moke et al., 2014). Cette cherté entrave encore son utilisation par les petits agriculteurs. Cette situation laisse donc envisager l'utilisation de techniques de fertilisations peu coûteuses et permettant une protection de l'environnement. De ce fait, la méthode alternative largement acceptée par les agriculteurs est l'utilisation des amendements organiques animaux ou végétaux (Soltner, 2003). Mais, leur utilisation doit être connue, maitrisée et raisonnée pour obtenir de bons rendements.

Face à toutes ces contraintes, nous avons trouvé nécessaire de mener une étude portant sur l'effet de quatre types de fertilisants sur la croissance et la productivité de deux génotypes de maïs (Zea mays L.) en cas d'un semis tardif au Centre-Nord de la Côte d'Ivoire (Korhogo). Elle a pour objectif d'évaluer l'influence des fumures utilisées sur le potentiel agronomique de ces génotypes de maïs cultivés en cas de contrainte hydrique et d'infertilité des sols. De façon spécifique, il s'est agi, (1) de déterminer les différentes phases phénologiques des variétés de maïs étudiées, (2) de caractériser le bilan climatique, (3) de comparer l'effet des fumures sur les paramètres de croissance et (4) d'analyser le rendement en fonction des différents traitements apportés. 


\section{MATÉRIEL ET MÉTHODES}

\section{Site expérimental et conduite de la culture}

L'essai a été conduit sur le site expérimental de l'Université Peleforo GON COULIBALY de Korhogo. Le sol du site expérimental est de type rouge sablo argileux. Les résultats de l'analyse des échantillons de sol dudit site sont consignés dans le Tableau 1. L'essai a été mis en place après une jachère constituée essentiellement d'Imperata cylindrica.

\section{Matériel végétal}

Le matériel végétal a été constitué de deux variétés locales de maïs. Il s'est agi d'une variété de maïs à grain de couleur noire et d'une autre, à grain de couleur violette. Ce sont des variétés de la ville de Katiola au Nord de la Côte d'Ivoire, respectivement appelées "nande n'kongon et nande worhor" (N'da et al., 2013). Ces variétés sont précoces, de longueur de cycle d'environ 90 jours. Elles ont un rendement moyen de 3 à 5 t/ha et un rendement potentiel pouvant atteindre $7 \mathrm{t} / \mathrm{ha}$ dans les conditions optimales de production (Yapi, 2017).

\section{Méthodes}

\section{Dispositif expérimental et traitements étudiés} Dispositif expérimental

Un dispositif expérimental en split-plot à quatre répétitions et deux facteurs a été utilisé. Le premier facteur, fertilisation, a été constitué de cinq niveaux, à savoir F0 qui correspond au témoin sans apport de fumure (Témoin); F1, l'engrais minéral (YaraMila Actyva $^{\mathrm{TM}}$ ); F2 constitué de la fiente de poulet (Poulet) ; F3, composé de la déjection de lapin (Lapin) et F4 correspondant à la déjection de bœuf (Bœuf). Le second facteur, variété, a été constitué de deux niveaux : V1, variété à grain noir notée $(\mathrm{N})$ et $\mathrm{V} 2$, variété à grain violet (V).

\section{Traitements étudiés}

Les combinaisons des fertilisants (facteur en sous-bloc) et des variétés (facteur en parcelles élémentaires) ont permis d'avoir un total de dix (10) traitements : F0V1 (NTémoin), F0V2 (V-Témoin), F1V1 (NYaraMila Actyva $\left.{ }^{\mathrm{TM}}\right)$, F1V2 (V- YaraMila Actyva $^{\mathrm{TM}}$ ), F2V1 (N-Poulet), F2V2 (V-
Poulet), F3V1 (N-Lapin), F3V2 (V-Lapin), F4V1 (N-Bœuf) et F4V2 (V-Bœuf).

\section{Fertilisation}

Les quantités des différents engrais apportés aux plants sont présentées dans le Tableau 2. Pour les déjections de bœuf, lapin et poulet, une quantité de $12,5 \mathrm{~kg}$ a été appliquée sur chaque grande parcelle avant le semis des grains de maïs, soit $6,25 \mathrm{~kg}$ par sous parcelle. Quant à l'engrais minéral YaraMila $\operatorname{Actyva}^{\mathrm{TM}}(\mathrm{F} 1)$, il a été enfoui à environ $5 \mathrm{~cm}$ de profondeur et à une distance de $5 \mathrm{~cm} \mathrm{du}$ poquet, aux doses de 100 à 150 et de 200 $\mathrm{kg} / \mathrm{ha}$ respectivement les $15^{\mathrm{è}}$ et $30^{\text {è jours après }}$ semis (JAS). Les résultats de l'analyse des échantillons de fumure sont présentés dans le Tableau 3. Les teneurs (\%) en, carbone organique, matière organique, azote total ainsi que le rapport $\mathrm{C} / \mathrm{N}$ après analyse des échantillons y sont consignés.

Comme pour le sol, le rapport $\mathrm{C} / \mathrm{N}$ est souvent utilisé pour prédire la stabilité d'une matière organique simple dans le sol. Une matière à faible $\mathrm{C} / \mathrm{N} \quad(4$ à 12$)$ va être rapidement minéralisée en fournissant beaucoup d'azote minéral. La dégradation d'une matière à fort $\mathrm{C} / \mathrm{N}(15$ à 20$)$ va à l'inverse provoquer l'immobilisation de l'azote du sol par les microorganismes.

\section{Mesures et observations}

Observations phénologiques

Les observations phénologiques ont concerné les dates de levée, d'initiation paniculaire (stade 5-6 feuilles, représentant la date à laquelle l'on obtient $50 \%$ du nombre total des feuilles au cours du cycle de développement des deux variétés de maïs), les dates de floraison (fleurs mâles) et de maturité des grains.

Les dates de semis et de levée ont permis de déterminer le temps d'émergence (délai d'émergence), celles de levée, de floraison mâle et de maturité ont permis d'établir les durées des phases végétative et reproductive pour chaque traitement. La longueur du cycle qui correspond à la date de maturité a également été déterminée. Pour chaque parcelle, les différents stades ont été considérés comme effectifs lorsque $50 \%$ des plants l'avaient atteint. 
Mesure des paramètres de croissance

Les mesures agro-morphologiques ont été faites sur 3 poquets pris au hasard sur les lignes internes de chaque parcelle. Ils ont été choisis de sorte à éviter ceux situés dans les bordures des parcelles élémentaires. Ces mesures ont été faites par quinzaine et ont porté sur la hauteur du plant (HP), le diamètre au collet du plant (DC), le nombre de feuilles vertes (NFV), et le nombre d'épis par plant (NEpis).

Analyse du rendement

Le rendement a été déterminé à la récolte sur six poquets situés au centre des parcelles et exprimé en tonnes par hectare (t/ha). Il a été calculé grâce au rapport du poids sec des grains par poquet et de la surface occupée par le poquet au sol.

Calcul de l'évapotranspiration de référence (ETO)

L'ETo journalière a été calculée à partir des températures maximum et minimum $\left({ }^{\circ} \mathrm{C}\right)$, l'humidité relative $(\%)$, la vitesse du vent $\left(\mathrm{m} . \mathrm{s}^{-1}\right)$ et l'insolation (heures). Ce calcul a été fait à l'aide du logiciel Cropwat version 8.0. Les données climatiques proviennent des prévisions de la Société World-WeatherOnline. Quant aux données pluviométriques, elles ont été obtenues grâce à un pluviomètre à lecture directe installé sur le site expérimental.

\section{Traitement et analyse statistique des données}

Le traitement des données des différents paramètres étudiés (la phénologie, les paramètres de croissance et le rendement) a été effectué à l'aide du tableur Excel. Ce tableur a également été utilisé pour le tracé des tableaux et des graphiques. L'analyse de la variance (ANOVA), a été effectuée avec le logiciel statistique STATISTIX version 8.1. Le test de Fisher Least Significant Différence (LSD) a été considéré, pour le classement des moyennes au seuil de $5 \%$ en cas de différence significative.

Tableau 1 : Résultats analytiques de 3 échantillons de sol du site expérimental.

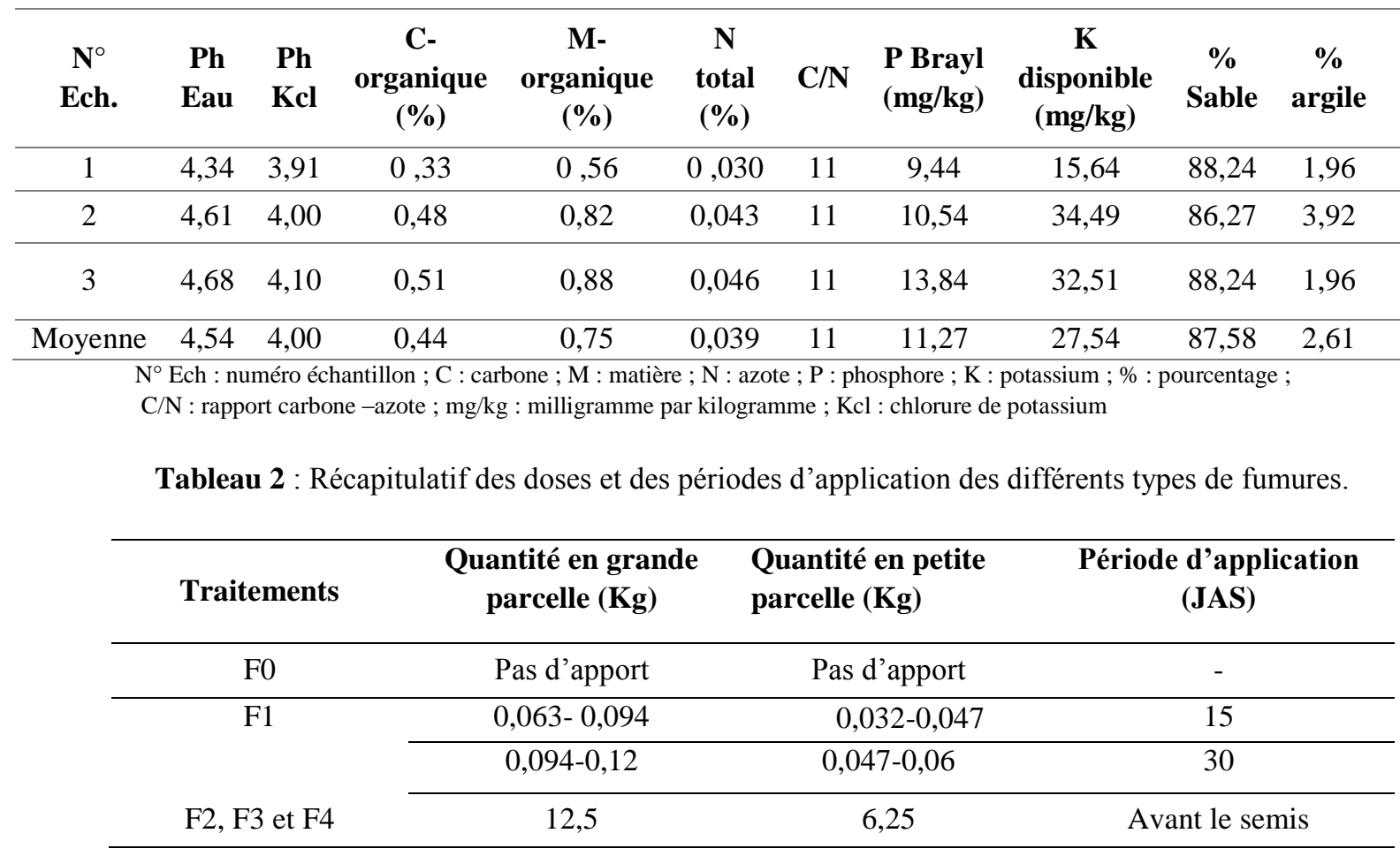


Tableau 3: Résultats analytiques des échantillons des fumures utilisées au cours de l'expérimentation.

\begin{tabular}{lcccc}
\hline Nature échantillon & Carbone (\%) & Matière organique (\%) & Azote total (\%) & C/N \\
\hline Dejection boeuf & 42,98 & 74,11 & 2,20 & 20 \\
\hline Dejection lapin & 36,43 & 62,81 & 1,48 & 24,5 \\
\hline Fiente poulet & 38,19 & 65,83 & 3,01 & 13 \\
\hline
\end{tabular}

$\mathrm{C} / \mathrm{N}$ : rapport carbone - azote $; \%$ : pourcentage

\section{RÉSULTATS}

\section{Caractérisation des phases phénologiques des variétés étudiées}

L'analyse de la variance n'a montré aucune différence entre les variétés et les traitements (Tableau 4), pour le délai d'émergence (4 jours), et de l'initiation paniculaire (16-18 jours). Par contre, des différences ont été observées au niveau des durées des phases végétative et reproductive et aussi, du cycle. Les durées de la phase végétative des plants des traitements V-Bœuf, V-Lapin, V-Témoin et V- YaraMila Actyva ${ }^{\mathrm{TM}}$ ont été équivalentes et les plus longues (53 Jours). Celles des plants des traitements $\mathrm{N}$ Bœuf et N-Poulet (49 Jours), ont été les plus courtes et les plants des traitements V- Poulet, $\mathrm{N}$-Lapin, N- Témoin et $\mathrm{N}$ - YaraMila Actyva $^{\mathrm{TM}}$, ont eu une durée de phase végétative intermédiaire de 51 Jours.

La durée de la phase reproductive, a été plus longue chez le traitement VYaraMila Actyva $^{\mathrm{TM}}$ avec un retard qui a varié entre 4 et 11 jours par rapport aux autres traitements. La durée de la phase reproductive la plus longue (41 jours) a été obtenue avec les plants du traitement V-YaraMila Actyva $^{\mathrm{TM}}$. Elle a été suivi de celles des plants des traitements N-témoin et $\mathrm{N}$ - YaraMila Actyva $^{\mathrm{TM}}$ (37 Jours) et la durée la plus courte comprise entre 30 à 32 jours, avec les plants des traitements N-bœuf, N-lapin, N-poulet, Vbœuf, V-lapin et V-poulet. Les durées de la phase reproductive et du cycle du traitement V-Témoin n'ont pu être déterminées car, les plantes de ce traitement n'ont pas produit d'épis. La durée du cycle des plants du traitement V- YaraMila Actyva ${ }^{\mathrm{TM}}$, a été la plus longue (98 jours) et celles des plants des traitements V-poulet, N-lapin, N-bœuf et Npoulet, les plus courtes (85 Jours chacun). Quant aux plants des autres traitements, ils ont présenté une valeur intermédiaire de 92 Jours.

\section{Bilans climatique et pluviométrique}

La Figure 1 représente l'évolution de l'évapotranspiration de référence et des pluies journalières durant le cycle de la culture des variétés de maïs étudiées. L'ETo (demande évaporative) a varié de $5,2 \mathrm{~mm} / \mathrm{j}$ à $5,8 \mathrm{~mm} / \mathrm{j}$ au cours de l'expérimentation. La quantité totale de pluie enregistrée au cours de l'expérimentation a été de $344 \mathrm{~mm}$. Du semis à la floraison, les résultats ont montré un excédent pluviométrique. Il a été enregistré $338 \mathrm{~mm}$ de pluie pour une valeur de ETo totale de $312,78 \mathrm{~mm}$. Entre la phase de floraison (57 JAS) et la fin de la phase de remplissage des graines, soit trois semaines après la floraison (78 JAS) de tous les traitements, $6 \mathrm{~mm}$ de pluie ont été enregistrés pour une ETo de $231,46 \mathrm{~mm}$, soit un déficit d'environ 37 fois inférieur à la demande évaporative. Une contrainte hydrique a donc été notée durant cette période.

\section{Effet des fertilisants sur les paramètres de croissance}

\section{Hauteur des plants}

L'évolution de la valeur moyenne de la hauteur des plants exprimée en centimètre $(\mathrm{cm})$, en fonction des traitements est présentée 
dans le Tableau 5. Le test statistique a montré une différence de hauteur moyenne entre les plants des différents traitements au cours de notre expérimentation, excepté le $50^{\mathrm{e}} \mathrm{JAS}$. Au

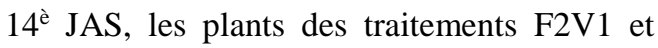
F2V2, ont enregistré les meilleures valeurs de hauteur comprises entre 13,16 et $14,08 \mathrm{~cm}$ et ceux du traitement F0V2, la hauteur la plus faible $(8,67 \mathrm{~cm})$. Les autres traitements ont donné des hauteurs intermédiaires oscillant entre 9,17 et $10,50 \mathrm{~cm}$. Au $35^{\mathrm{e}}$ JAS. La même tendance a été observée avec des valeurs moyennes comprises entre 58,33 et $67 \mathrm{~cm}$, pour les plants des traitements F2V2 et F2V1; $18,58 \mathrm{~cm}$ pour ceux du traitement F0V2 et des hauteurs intermédiaires de $29,92 \mathrm{~cm}$ à $50 \mathrm{~cm}$ pour les plants des autres traitements. Au $64^{\mathrm{e}}$ JAS, après la floraison, avec des valeurs moyennes comprises entre 201,83 et 220,17 $\mathrm{cm}$, les plants des traitements F2V1, F2V2, F4V1, F4V2, F3V1 et F3V2, ont enregistré les hauteurs de plants les plus importants. Les plants du traitement F0V2, ont affiché les hauteurs les plus faibles $(115,67 \mathrm{~cm})$ et ceux des traitements F1V1, F1V2 et F0V1, des moyennes de hauteur intermédiaires (114 $198,83 \mathrm{~cm}$ ).

\section{Diamètre au collet des plants}

Pour tous les traitements étudiés, le diamètre au collet a augmenté du $14^{\grave{e}}$ au $85^{\text {è }}$ jour. (Tableau 6). Au 92 $2^{\mathrm{è}} \mathrm{JAS}$, le diamètre au collet des plants des traitements avec fumure ont présenté des valeurs moyennes statistiquement équivalentes $(14,09-15,79$ $\mathrm{cm})$ et supérieures à celles des traitements sans fumure, F0V1 et F0V2 $(6,80-9.55 \mathrm{~cm})$.

\section{Nombre de feuilles vertes par plant}

Nos résultats ont montré que le nombre de feuilles vertes a augmenté dans tous les traitements jusqu'à $50 \mathrm{JAS}$, puis, il a connu une chute (Tableau 7). À 35 JAS, l'apport de fumure a eu tendance à doubler le nombre de feuilles vertes. La variété à grain noir, cultivée sans fumure (F0V1) a présenté le même nombre de feuilles que les traitements avec fumure et cette tendance a été maintenue

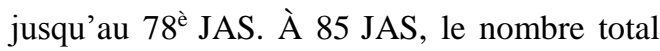
de feuilles vertes a commencé à diminuer et à 92 JAS, une baisse drastique a été constatée chez tous les plants de tous les traitements.

\section{Nombre d'épis}

Les résultats du test statistique ont montré une différence entre les traitements de 64 à 92 JAS pour le nombre d'épis (Tableau 8). Au 64 Jè JAS, soit à l'épiaison, le plus grand nombre d'épis $(1,17)$, a été observé avec les plants des traitements F2V2, F4V1 et F1V1 et le plus faible $(0,25)$, avec les plants du traitement F0V2. Aux dates de 78 JAS et 85 JAS, le nombre d'épis a été semblable pour tous les plants de tous les traitements, puis à 92 JAS, en fin de cycle, une différence a été observée entre les traitements. Les plants des traitements F4V1, F3V1, F4V2, F3V2 et F1V2 ont affiché un nombre d'épis compris entre 1,25 - 1,33 épis en moyenne, tandis que chez les plants du traitement F0V2, il a été de 0,75 épi.

\section{Effet des fertilisants sur le rendement}

Il a été révélé des différences significatives entre les effets des différents traitements sur le rendement des plants (Figure 2). La variété à grain noir a obtenu de meilleurs rendements (2 à $2,8 \mathrm{t}$ ) par rapport à la variété à grain violet ( 1 à 1,8 t) quelle que soit la fumure. Les fumures organiques et l'engrais minéral ont amélioré les valeurs de rendement des différentes variétés relativement aux témoins. Chez la variété à grain noir, les rendements obtenus grâce à l'engrais minéral YaraMila Actyva ${ }^{\mathrm{TM}}$, les fumures (déjections de lapin, de bœuf et fiente de poulet) ont permis respectivement d'obtenir 5 fois et 6 fois le rendement obtenu par le témoin. 
Tableau 4: Suivi des phases phénologiques (en Jour) des variétés de maïs en fonction des traitements.

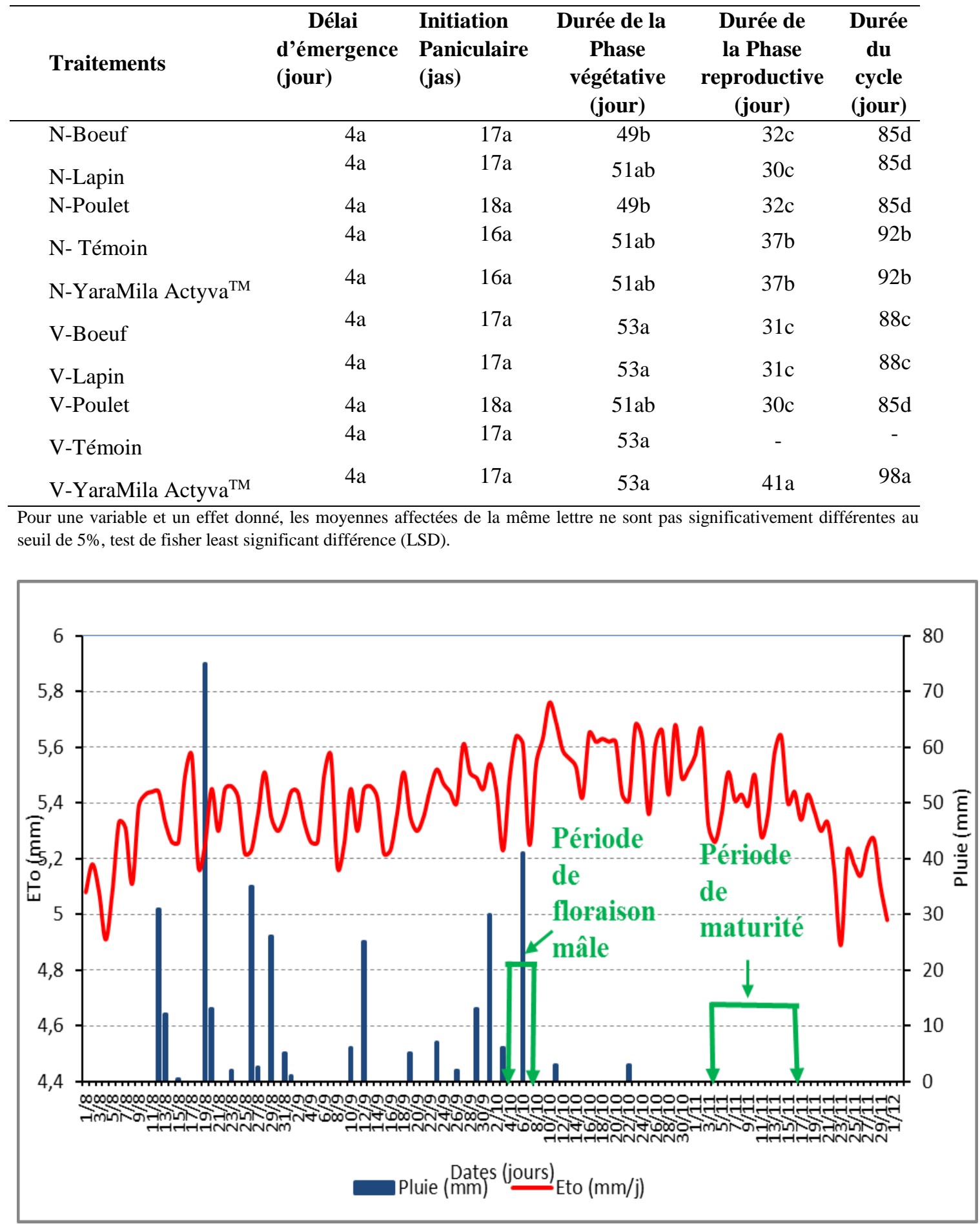

Figure 1 : Représentations graphiques de ETo $(\mathrm{mm})$ et des précipitations $(\mathrm{mm})$ en fonction des jours. Eto : évapotranspiration de référence. 
Tableau 5 : Hauteur des plants des différents traitements durant l'expérimentation.

\begin{tabular}{clllll}
\hline Traitements & $\mathbf{1 4}$ jas & $\mathbf{2 1}$ jas & $\mathbf{3 5}$ jas & $\mathbf{5 0}$ jas & $\mathbf{6 4}$ jas \\
\hline F2V1 & $13,16 \mathrm{ab}$ & $24,83 \mathrm{a}$ & $67,00 \mathrm{a}$ & $195,83 \mathrm{a}$ & $225,17 \mathrm{a}$ \\
F2V2 & $14,08 \mathrm{a}$ & $22,92 \mathrm{ab}$ & $58,33 \mathrm{ab}$ & $153,17 \mathrm{a}$ & $218,67 \mathrm{a}$ \\
F4V1 & $9,17 \mathrm{bc}$ & $19,33 \mathrm{abc}$ & $41,58 \mathrm{bc}$ & $143,50 \mathrm{a}$ & $207,83 \mathrm{a}$ \\
F3V1 & $10,50 \mathrm{abc}$ & $17,83 \mathrm{bcd}$ & $48,75 \mathrm{abc}$ & $161,58 \mathrm{a}$ & $203,83 \mathrm{a}$ \\
F1V1 & $9,25 \mathrm{bc}$ & $13,33 \mathrm{~d}$ & $29,92 \mathrm{~cd}$ & $119,08 \mathrm{a}$ & $189,83 \mathrm{ab}$ \\
F4V2 & $9,25 \mathrm{bc}$ & $16,83 \mathrm{~cd}$ & $33,42 \mathrm{~cd}$ & $97,67 \mathrm{a}$ & $201,83 \mathrm{a}$ \\
F3V2 & $10,08 \mathrm{abc}$ & $16,50 \mathrm{~cd}$ & $50,00 \mathrm{abc}$ & $127,25 \mathrm{a}$ & $210,17 \mathrm{a}$ \\
F1V2 & $10,17 \mathrm{abc}$ & $17,08 \mathrm{bcd}$ & $32,83 \mathrm{~cd}$ & $95,50 \mathrm{a}$ & $176,25 \mathrm{ab}$ \\
F0V1 & $10,00 \mathrm{abc}$ & $15,33 \mathrm{~cd}$ & $32,50 \mathrm{~cd}$ & $80,25 \mathrm{a}$ & $144,00 \mathrm{bc}$ \\
F0V2 & $8,67 \mathrm{c}$ & $13,08 \mathrm{~d}$ & $18,58 \mathrm{~d}$ & $66,25 \mathrm{a}$ & $115,67 \mathrm{c}$
\end{tabular}

Pour une variable et un effet donné, les moyennes affectées de la même lettre ne sont pas significativement différentes au seuil de $5 \%$, test de fisher least significant différence (LSD).

Tableau 6 : Diamètre moyen au collet des plants des différents traitements durant l'expérimentation.

\begin{tabular}{llllllllll}
\hline Traitements & $\mathbf{1 4}$ jas & $\mathbf{2 1}$ jas & $\mathbf{3 5}$ jas & $\mathbf{5 0}$ jas & $\mathbf{6 4}$ jas & $\mathbf{7 1}$ jas & $\mathbf{7 8}$ jas & $\mathbf{8 5}$ jas & $\mathbf{9 2}$ jas \\
\hline F2V1 & $2,88 \mathrm{a}$ & $5,31 \mathrm{a}$ & $15,15 \mathrm{a}$ & $17,18 \mathrm{a}$ & $17,14 \mathrm{a}$ & $16,90 \mathrm{a}$ & $16,76 \mathrm{a}$ & $11,15 \mathrm{ab}$ & $14,63 \mathrm{a}$ \\
F2V2 & $2,69 \mathrm{a}$ & $4,98 \mathrm{~b}$ & $13,50 \mathrm{ab}$ & $17,22 \mathrm{a}$ & $16,65 \mathrm{a}$ & $16,86 \mathrm{a}$ & $16,73 \mathrm{a}$ & $16,61 \mathrm{a}$ & $15,77 \mathrm{a}$ \\
F4V1 & $2,45 \mathrm{ab}$ & $4,10 \mathrm{abc}$ & $10,42 \mathrm{bcd}$ & $16,18 \mathrm{a}$ & $15,56 \mathrm{ab}$ & $15,72 \mathrm{ab}$ & $14,97 \mathrm{ab}$ & $15,38 \mathrm{ab}$ & $14,32 \mathrm{a}$ \\
F3V1 & $2,36 \mathrm{ab}$ & $3,97 \mathrm{abdc}$ & $10,78 \mathrm{bc}$ & $16,20 \mathrm{a}$ & $15,71 \mathrm{a}$ & $18,80 \mathrm{a}$ & $15,77 \mathrm{a}$ & $15,26 \mathrm{ab}$ & $14,09 \mathrm{a}$ \\
F1V1 & $2,25 \mathrm{ab}$ & $2,47 \mathrm{de}$ & $6,84 \mathrm{de}$ & $15,16 \mathrm{a}$ & $15,95 \mathrm{a}$ & $16,76 \mathrm{a}$ & $15,98 \mathrm{a}$ & $15,55 \mathrm{ab}$ & $14,69 \mathrm{a}$ \\
F4V2 & $2,48 \mathrm{ab}$ & $1,82 \mathrm{cde}$ & $6,75 \mathrm{de}$ & $14,06 \mathrm{ab}$ & $15,48 \mathrm{ab}$ & $15,62 \mathrm{ab}$ & $15,91 \mathrm{a}$ & $15,66 \mathrm{a}$ & $15,14 \mathrm{a}$ \\
F3V2 & $2,12 \mathrm{ab}$ & $3,26 \mathrm{cde}$ & $9,74 \mathrm{bcd}$ & $15,22 \mathrm{a}$ & $15,78 \mathrm{a}$ & $16,09 \mathrm{a}$ & $15,57 \mathrm{a}$ & $15,91 \mathrm{a}$ & $15,79 \mathrm{a}$ \\
F1V2 & $1,84 \mathrm{~b}$ & $3,5 \mathrm{bcde}$ & $6,87 \mathrm{cde}$ & $14,30 \mathrm{ab}$ & $15,27 \mathrm{ab}$ & $15,16 \mathrm{ab}$ & $15,45 \mathrm{ab}$ & $15,80 \mathrm{a}$ & $15,29 \mathrm{a}$ \\
F0V1 & $2,18 \mathrm{ab}$ & $2,6 \mathrm{cde}$ & $6,03 \mathrm{e}$ & $12,23 \mathrm{ab}$ & $11,13 \mathrm{bc}$ & $11,37 \mathrm{~b}$ & $10,85 \mathrm{bc}$ & $11,14 \mathrm{~b}$ & $9,55 \mathrm{~b}$ \\
F0V2 & $1,84 \mathrm{~b}$ & $2,31 \mathrm{e}$ & $3,34 \mathrm{e}$ & $8,54 \mathrm{~b}$ & $7,276 \mathrm{c}$ & $7,25 \mathrm{c}$ & $7,50 \mathrm{c}$ & $6,84 \mathrm{c}$ & $6,80 \mathrm{~b}$ \\
\hline
\end{tabular}

Pour une variable et un effet donné, les moyennes affectées de la même lettre ne sont pas significativement différentes au seuil de 5\%, test de fisher least significant différence (LSD).

Tableau 7 : Nombre de feuilles vertes par plant des différents traitements durant l'expérimentation.

\begin{tabular}{llllllllll}
\hline Traitements & $\mathbf{1 4}$ jas & $\mathbf{2 1}$ jas & $\mathbf{3 5}$ jas & $\mathbf{5 0}$ jas & $\mathbf{6 4}$ jas & $\mathbf{7 1}$ jas & $\mathbf{7 8}$ jas & $\mathbf{8 5}$ jas & $\mathbf{9 2}$ jas \\
\hline F2V1 & $4,83 \mathrm{a}$ & $7,08 \mathrm{a}$ & $10,08 \mathrm{a}$ & $10,08 \mathrm{a}$ & $8,75 \mathrm{a}$ & $8,50 \mathrm{a}$ & $7,42 \mathrm{a}$ & $3,67 \mathrm{c}$ & $0,50 \mathrm{a}$ \\
F2V2 & $4,42 \mathrm{ab}$ & $6,83 \mathrm{a}$ & $9,50 \mathrm{a}$ & $9,50 \mathrm{a}$ & $9,08 \mathrm{a}$ & $8,67 \mathrm{a}$ & $8,25 \mathrm{a}$ & $6,42 \mathrm{ab}$ & $3,17 \mathrm{a}$ \\
F4V1 & $4,17 \mathrm{ab}$ & $5,92 \mathrm{abc}$ & $8,25 \mathrm{abcd}$ & $10,00 \mathrm{a}$ & $9,17 \mathrm{a}$ & $8,83 \mathrm{a}$ & $8,17 \mathrm{a}$ & $5,83 \mathrm{abc}$ & $1,33 \mathrm{a}$ \\
F3V1 & $4,17 \mathrm{ab}$ & $6,50 \mathrm{ab}$ & $8,75 \mathrm{ab}$ & $10,08 \mathrm{a}$ & $9,00 \mathrm{a}$ & $9,17 \mathrm{a}$ & $8,58 \mathrm{a}$ & $5,92 \mathrm{abc}$ & $1,75 \mathrm{a}$ \\
F1V1 & $4,08 \mathrm{ab}$ & $5,00 \mathrm{~cd}$ & $7,25 \mathrm{bcd}$ & $9,83 \mathrm{a}$ & $9,50 \mathrm{a}$ & $9,00 \mathrm{a}$ & $8,08 \mathrm{a}$ & $5,67 \mathrm{bc}$ & $1,67 \mathrm{a}$ \\
F4V2 & $3,83 \mathrm{~b}$ & $5,33 \mathrm{bcd}$ & $6,67 \mathrm{bcde}$ & $9,17 \mathrm{a}$ & $9,33 \mathrm{a}$ & $8,67 \mathrm{a}$ & $7,83 \mathrm{a}$ & $6,17 \mathrm{abc}$ & $3,50 \mathrm{a}$ \\
F3V2 & $4,08 \mathrm{ab}$ & $5,92 \mathrm{abc}$ & $8,50 \mathrm{abc}$ & $9,08 \mathrm{a}$ & $9,25 \mathrm{a}$ & $9,08 \mathrm{a}$ & $8,67 \mathrm{a}$ & $6,83 \mathrm{ab}$ & $4,25 \mathrm{a}$ \\
F1V2 & $3,75 \mathrm{~b}$ & $5,08 \mathrm{~cd}$ & $6,58 \mathrm{cde}$ & $8,92 \mathrm{a}$ & $9,58 \mathrm{a}$ & $9,58 \mathrm{a}$ & $9,25 \mathrm{a}$ & $8,33 \mathrm{a}$ & $5,42 \mathrm{a}$ \\
F0V1 & $4,08 \mathrm{ab}$ & $5,08 \mathrm{~cd}$ & $6,33 \mathrm{de}$ & $8,83 \mathrm{a}$ & $7,83 \mathrm{ab}$ & $7,92 \mathrm{a}$ & $7,50 \mathrm{a}$ & $5,50 \mathrm{bc}$ & $2,67 \mathrm{a}$ \\
F0V2 & $4,00 \mathrm{~b}$ & $4,67 \mathrm{~d}$ & 4,58 & $6,00 \mathrm{~b}$ & $5,75 \mathrm{~b}$ & $5,33 \mathrm{~b}$ & $4,92 \mathrm{~b}$ & $3,83 \mathrm{c}$ & $1,92 \mathrm{a}$ \\
\hline
\end{tabular}

Pour une variable et un effet donné, les moyennes affectées de la même lettre ne sont pas significativement différentes au seuil de 5\%, test de fisher least significant différence (LSD). 
Tableau 8 : Nombre d'épis par plant des différents traitements durant l'expérimentation.

\begin{tabular}{clllll}
\hline Traitements & $\mathbf{6 4}$ jas & $\mathbf{7 1}$ jas & $\mathbf{7 8}$ jas & $\mathbf{8 5}$ jas & $\mathbf{9 2}$ jas \\
\hline F2V1 & $1,17 \mathrm{a}$ & $1,00 \mathrm{abc}$ & $1,08 \mathrm{a}$ & $1,08 \mathrm{a}$ & $1,08 \mathrm{ab}$ \\
F2V2 & $1,00 \mathrm{abc}$ & $1,00 \mathrm{abc}$ & $1,08 \mathrm{a}$ & $1,08 \mathrm{a}$ & $1,08 \mathrm{ab}$ \\
F4V1 & $1,17 \mathrm{a}$ & $1,33 \mathrm{a}$ & $1,25 \mathrm{a}$ & $1,25 \mathrm{a}$ & $1,25 \mathrm{a}$ \\
F3V1 & $1,25 \mathrm{ab}$ & $1,33 \mathrm{a}$ & $1,33 \mathrm{a}$ & $1,33 \mathrm{a}$ & $1,33 \mathrm{a}$ \\
F1V1 & $1,17 \mathrm{a}$ & $1,17 \mathrm{ab}$ & $1,25 \mathrm{a}$ & $1,25 \mathrm{a}$ & $0,92 \mathrm{ab}$ \\
F4V2 & $1,08 \mathrm{abc}$ & $1,25 \mathrm{a}$ & $1,25 \mathrm{a}$ & $1,25 \mathrm{a}$ & $1,25 \mathrm{a}$ \\
F3V2 & $0,92 \mathrm{bc}$ & $1,17 \mathrm{ab}$ & $1,25 \mathrm{a}$ & $1,25 \mathrm{a}$ & $1,25 \mathrm{a}$ \\
F1V2 & $0,67 \mathrm{~cd}$ & $1,25 \mathrm{a}$ & $1,33 \mathrm{a}$ & $1,33 \mathrm{a}$ & $1,33 \mathrm{a}$ \\
F0V1 & $0,83 \mathrm{bc}$ & $0,83 \mathrm{bc}$ & $1,08 \mathrm{a}$ & $1,08 \mathrm{a}$ & $1,08 \mathrm{ab}$ \\
F0V2 & $0,25 \mathrm{~d}$ & $0,66 \mathrm{c}$ & $0,75 \mathrm{a}$ & $0,75 \mathrm{a}$ & $0,75 \mathrm{~b}$ \\
\hline
\end{tabular}

Pour une variable et un effet donné, les moyennes affectées de la même lettre ne sont pas significativement différentes au seuil de 5\%, test de fisher least significant différence (LSD).

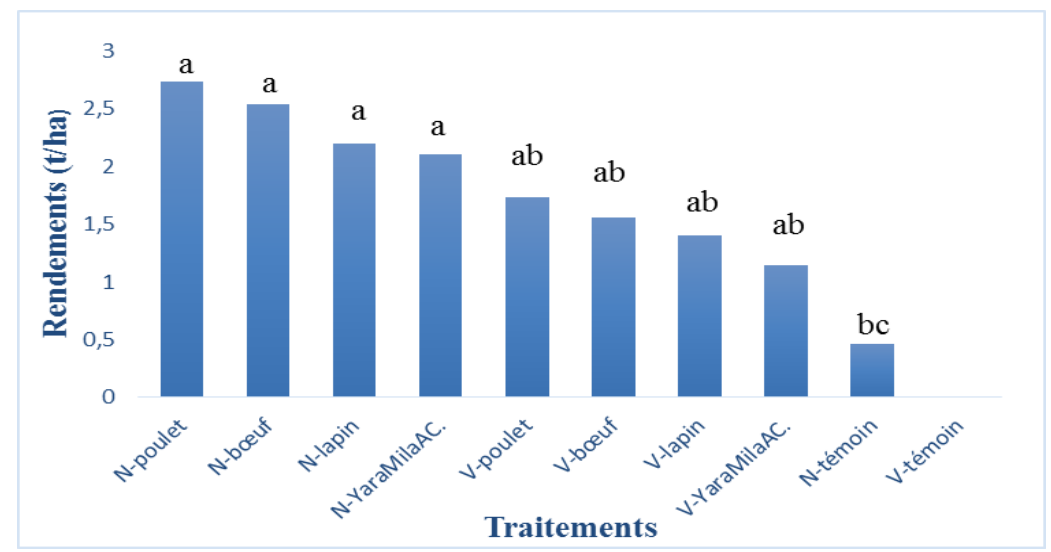

Figure 2 : Rendement des deux variétés de maïs étudiées.

Pour une variable et un effet donné, les moyennes affectées de la même lettre ne sont pas significativement différentes au seuil de 5\%, test de fisher least significant différence (LSD).

\section{DISCUSSION}

Les résultats obtenus concernant le délai d'émergence et l'initiation paniculaire, n'ont montré aucune différence entre les différents traitements. Quel que soit le traitement appliqué, il a été de 4 JAS pour le délai d'émergence. Ce résultat est corroboré par celui de (Diatta, 2007) qui a montré que la levée est constante quelle que soit la variété de maïs et la levée est effective au bout de 4 JAS. En effet, la germination subit l'effet de certains facteurs extérieurs, comme la disponibilité en eau, la température, qui ont un impact direct sur le métabolisme et les caractéristiques internes de la graine telles que la taille et la quantité de réserves ou de son génome (Anzala, 2006). Or, à ce stade de notre expérimentation, ces contraintes n'étaient pas effectives. Aussi, au niveau de l'initiation paniculaire, aucune différence n'a été constatée entre les traitements malgré son déroulement pendant une pause pluviométrique au cours de notre étude. En effet, c'est au cours de cette phase que les organes reproducteurs sont initiés, la taille des grains et le nombre de rangées de grains par épis, sont définis. Elle est sensible au déficit hydrique et en éléments nutritifs. Mais, selon de nombreux auteurs (Hema, 2000 ; Lafitte, 2002 ; Tine, 2008), les phases au cours desquelles les conséquences d'un stress hydrique sont les plus graves sont d'une part le semis et les deux semaines qui le suivent et d'autre part, les deux semaines qui précèdent et les deux qui suivent la floraison. 
La durée des phases végétatives et reproductives des variétés étudiées a été influencée par les différentes fumures. Ainsi, comparativement à la variété à grain violet, la variété à grain noir a présenté des durées de phase végétative plus courtes, notamment avec les plants ayant reçu la fiente de poulet et la déjection de bœuf. Cela pourrait s'expliquer par la disponibilité des éléments minéraux contenus dans ces fumures. En effet, plusieurs comportements sont distingués quant à la phase de minéralisation plus rapide de l'azote organique. L'azote organique des engrais organiques type fientes ou fumiers de volailles se minéralise rapidement par rapport aux autres fumures. Cette observation est confirmée par les résultats analytiques des fumures organiques utilisées dans notre étude. Ces résultats ont montré un faible rapport $\mathrm{C} / \mathrm{N}$ de cette fumure par rapport aux autres fumures organiques. Le rapport $\mathrm{C} / \mathrm{N}$ d'une matière renseigne sur sa vitesse de minéralisation et la faible valeur de ce rapport pourrait traduire la relative rapide minéralisation de l'azote et par conséquent sa disponibilité (Aké et al., 2018). Les fumiers de volailles présentent donc un grand potentiel pour l'amélioration de la disponibilité des éléments nutritifs du sol et de la fourniture de nutriments nécessaires à la culture du maïs (Nyembo et al., 2014). De plus, la minéralisation de la fiente de poulet favoriserait une disponibilité en azote totale et en phosphore assimilable dans le sol (Dean et al., 2000). Ce phosphore pourrait expliquer le raccourcissement de la phase végétative chez la variété à grain noir, car, il serait un facteur de précocité comme signalé par Kati (2012). De ce fait, il stimule la levée, raccourcit le cycle végétatif, accélère la maturation et la constitution des réserves. En ce qui concerne la différence de la durée de la phase reproductive des plants des différents traitements, elle découlerait de la différence observée au niveau des phases végétatives, mais surtout, du déficit hydrique enregistré en fin de cycle. Comme indiqué par Kocou (2012), chez les céréales, la durée de la phase reproductive reste constante quelle que soit la variété. Pour le cas du riz, elle dure pratiquement une trentaine de jours et c'est la période pendant laquelle la plante a les besoins les plus élevés en éléments nutritifs et en eau. La conséquence de ces observations a donc été les différences de durées du cycle végétatif.

Les effets des différents fertilisants sur les paramètres de croissance, ont été remarquables. Les plants ayant reçu les fumures organiques et minérales ont présenté une forte vigueur de croissance en hauteur ainsi que du diamètre au collet, en particulier, celles traitées avec la fiente de poulet quelle que soit la variété. Ce constat met en exergue la disponibilité des éléments minéraux dans les engrais organiques pour la croissance et le développement des cultures en plein champ et comme pour le maïs par des apports organominéraux (Muyayabantu et al., 2012). De plus, les résultats obtenus chez les plants ayant reçu la fiente de poulet pourraient s'expliquer par le rôle primordial que joue le phosphore assimilable libéré dans le sol par cette fumure dans la croissance et le développement, ainsi que dans le métabolisme des plantes et le transport de l'énergie (Ouédraogo et al., 2014). Ainsi, le niveau bas de réserves nutritives observé lors de l'analyse des échantillons du sol des parcelles témoins, expliquerait la cause majeure de la mauvaise performance de la croissance en hauteur du mais observée sur celles-ci.

Les plants ont émis la totalité de leur feuille juste avant l'apparition de la fleur mâle, quel que soit le traitement. Ce résultat est corroboré par ceux obtenus par Siéné et al. (2018), qui ont montré que chez le maïs, l'émission des feuilles s'arrêtent peu de jours avant l'apparition de la panicule mâle pendant que la tige de la plante continue sa croissance. De même, selon Diatta (2007), un stress hydrique appliqué en phase reproductive n'affecte que le rendement en grains, car, il n'y a plus d'émission foliaire et que la plante a atteint son nombre maximum de feuilles. En effet, chez le maïs, la senescence demeure sous l'influence $\mathrm{du}$ fonctionnement reproductif (Borras et al., 2003). Le stress hydrique ne peut alors qu'accélérer la sénescence foliaire. Ces observations corroborent ainsi, le fait que la fumure fiente de poulet favoriserait une bonne croissance ainsi qu'un bon développement du maïs. En effet, cette contrainte a été caractérisée par la 
variation des quantités de pluie reçues au cours du cycle de culture. Du semis à la floraison (du 53 JAS au 57 JAS), elles ont été de $338 \mathrm{~mm}$. Une pause pluviométrique a été observée à partir de cette période jusqu'à la fin de la phase de remplissage des grains, soit trois (3) semaines après la floraison. Ainsi, la demande évaporative (ETo) a été importante et de l'ordre de 5,44 $\mathrm{mm}$. D'après Hoopen et Maïga (2012), le maïs est très sensible au stress (contrainte) d'humidité (pénurie d'eau) à ce stade étant donné ses besoins plus importants en eau (jusqu'à $10 \mathrm{~mm}$ par jour dans des conditions très chaudes et sèches). Nos conditions de culture auraient donc influencé la productivité des variétés de maïs étudiées.

$\mathrm{Du}$ point de vue du rendement des cultures, les fumures organiques et l'engrais minéral ont amélioré les valeurs de rendement des différentes variétés relativement aux témoins. Selon Mulaji (2011), l'une des voies prometteuses pour accroitre la production agricole en milieu paysan, consiste à apporter aux sols différents types de matières organiques et des engrais minéraux de sorte à accroître la disponibilité des éléments nutritifs dans le sol pour les plantes. De plus, le rendement obtenu avec les plants de la variété à grain noir fertilisés avec la fiente de poulet (2,8 t/ha) se rapproche de celui obtenu $(3,039$ t/ha) par Gomgnimbou et al. (2019) avec une fumure organique constituée de fiente de poulet à la dose de $5 \mathrm{t} / \mathrm{ha}$. Le faible rendement obtenu au niveau des plants témoins pourrait s'expliquer par la pauvreté du sol en éléments nutritifs couplé au déficit hydrique enregistré au cours de la phase reproductive. Ce déficit hydrique pouvant entrainer à lui seul, une perte de 20 à 25 pourcent du rendement et atteindre 60 pourcent si le stress survient à la période critique (20 jours avant la floraison et se poursuit 10 jours après). Pendant cette période, le maïs mobilise 45 pourcent de ses besoins en eau. Le processus de remplissage des grains est alors ralenti voire bloqué (Hiema, 2005). Aussi, nos résultats ont-ils montré que les valeurs de rendement obtenues avec la variété à grain noir (environ 3 t/ha), ont été proches du rendement moyen obtenu chez ladite variété (3 à $5 \mathrm{t} / \mathrm{ha}$ ), tandis que celles obtenues avec la variété à grain violet ont été plus faible quelle que soit la fumure utilisée. Ces résultats pourraient s'expliquer par son cycle qui a été plus long quel que soit le traitement. Cette variété aurait donc été plus sensible et affectée par le déficit hydrique de fin de cycle combiné aux valeurs élevées de demande évaporative enregistrées au cours de la phase reproductive. En effet, selon Yang et Zhang (2006) et Aslani et Mehrvar (2012), les semis tardifs des céréales en conditions de déficit hydrique et de fortes températures en phase de remplissage des grains provoquent une augmentation de la proportion des grains de petite taille et immatures.

\section{Conclusion}

L'étude menée dans le but d'évaluer l'effet de quatre types de fertilisant sur la croissance et la productivité de deux génotypes de maïs (Zea mays L.) après un semis tardif au Centre-Nord de la Côte d'Ivoire, a fourni des résultats prometteurs. Elle a permis de mettre en évidence l'impact des fumures utilisées sur la productivité des variétés de maïs étudiées. Concernant la phénologie des variétés étudiées, aucun effet des fumures n'a été observé sur le délai d'émergence et l'initiation paniculaire. Par contre, elles ont affecté les durées des phases végétative et reproductive, et par conséquent la durée du cycle. La variété à graine violette a été plus affectée par le déficit hydrique au cours de la phase reproductive par rapport à la variété à graine noire. Les différents fertilisants organiques et minéraux ont différemment influencé les paramètres de croissance et le rendement des variétés étudiées. Concernant les paramètres de croissance des plants, la fumure fiente de poulet s'est nettement distinguée des autres. Elle a accéléré l'élongation des tiges, le rythme d'émission des feuilles, de même que la croissance du diamètre au collet des plants. Ce fort développement végétatif provoqué par cette fumure a eu pour conséquence une sénescence rapide des feuilles face au déficit hydrique enregistré de la floraison jusqu'en fin de cycle. La fumure fiente de poulet a aussi affiché, une tendance à réduire le cycle de culture. Au niveau du rendement, celui obtenu avec la variété à grain noir a été supérieur à celui de la variété à grain violet 
quelle que soit la fumure. De plus, les quatre types de fertilisant ont amélioré le rendement par rapport aux témoins indépendamment de la variété. Ils ont permis de produire quatre (5) à six (6) fois plus qu'en culture sans apport de fumure. Les fumures fiente de poulet et déjection de bœuf ont été les plus efficaces dans nos conditions de culture. Elles ont permis d'obtenir un rendement qui est égal à 6 fois celui obtenu avec le témoin de la variété à grain noir malgré la contrainte hydrique enregistré en fin de cycle. Leur utilisation en culture du maïs, pourrait constituer une alternative face aux conséquences drastiques du changement climatique.

\section{CONFLIT D'INTERETS}

Les auteurs attestent qu'il n'existe aucun conflit d'intérêts entre eux ou autre partie au sujet de cet article.

\section{CONTRIBUTIONS DES AUTEURS}

LACS a rédigé le protocole d'expérimentation, contribué à la mise en place de l'essai, à la vérification et à l'exploitation des données (analyses statistiques) ainsi qu'à la rédaction de la première version du manuscrit. MD et MST ont veillé à la conformité des résultats et contribué à la rédaction du manuscrit. MC, TVFN et MK ont participé à la mise en place de l'essai, au suivi et à la récolte des données.

\section{REMERCIEMENTS}

Nos remerciements vont à l'endroit de Docteur OUATTARA Owélé enseignantchercheur à l'Université Peleforo GON COULIBALY qui a mis à notre disposition les semences des variétés de maïs étudiées. Nos gratitudes vont également à l'endroit monsieur TUO Yalamoussa, Docteur en entomologie agricole à l'Université Peleforo GON COULIBALY pour l'aide qu'il nous a apporté lors des traitements phytosanitaires des parcelles.

\section{RÉFÉRENCES}

Akanvou L, Akanvou R, Kouamé A, Diarrassouba L. 2006. Fiche technique «Bien cultiver le mais en Côte d'Ivoire ». Centre National de Recherche Agronomique, $4 \mathrm{p}$.
Aké HTB, Bi Tra T, Dogbo OD. 2018. Caractéristiques physico-chimiques des composts à base de sous-produits de ferme de Songon en Côte d'Ivoire. Int. J. Biol. Chem. Sci. 12(1): 596-609. DOI : https://dx.doi.org/10.4314/ijbcs.v12i1.45

Amigues JP, Debaeke P, Itier B, Lemaire G, Seguin B, Tardieu F, Thomas A. 2006. Sécheresse et agriculture. Réduire la vulnérabilité de l'agriculture à un risque accru de manque d'eau. Expertise scientifique collective. INRA, Paris, 72 p.

Anzala FJ. 2006. Contrôle de la vitesse de germination chez le maïs (Zea mays) : étude de la voie de biosynthèse des acides aminés issus de l'aspartate et recherche de QTLs. Thèse de doctorat, spécialité : biologie cellulaire et moléculaire végétale, Université d'Angers, France, p.147.

Aslani F, Mehrvar MR. 2012. Responses of wheat genotypes as affected by different sowing date. Asian Journal of Agricultural Sciences, 4(1): 72-74. DOI : https://maxwellsci.com/jp/abstract.phpjid /AJAS\&no/17012

Assié KH, Angui KT, Tamia AJ. 2008. Effet de la mise en culture et des contraintes naturelles sur quelques propriétés physiques d'un sol ferralitique au Centre-Ouest de la Côte d'Ivoire: conséquences sur la dégradation des sols. Eur. J. Sci. Res., 23:149-166. DOI : https://www.researchgate.net/publication 1285936822

Baboy LL, Kidinda KL, Kilumba KM, Langunu S, Mazinga KM, Tshipama TD, Nyembo KL. 2015. Influence du semis tardif sur la croissance et le rendement du soja (Glycine max Merril) cultivé sous différents écartements à Lubumbashi, RD Congo. International Journal of Innovation and Applied Studies, 12(1): 104-109. DOI: https://www.academia.edu/18469611

Boone PS, Charles JD, Wanzie RL. 2008. Évaluation sous régionale de la chaîne de valeurs du maïs, rapport technique ATP $\mathrm{n}^{\circ} 1$. Bethesda, MD: projet ATP, Abt Associates Inc. 
Borras L, Maddonni GA, Otegui ME. 2003. Leaf senescence in maize hybrids: plant population, row spacing and kernel set effects. Field Crops Res., 82(1): 13-26. DOI: $10.1016 / \mathrm{S} 0378-4290(03) 00002-9$

Dean DS, Earl CS, Raymond EK. 2000. Irrigation management for corn in the Northern Great Plains, USA. Irrig. Sci., 19: 107-114. DOI: https://link.springer.com/article/10.1007/ PL00006709

Diatta J. 2007. Caractérisation du développement physiologique, des biomasses et des surfaces foliaires de deux variétés typiques de maïs (Zea mays L.) : contribution au paramétrage d'un modèle de simulation du développement des cultures pour la prévision agricole au Sénégal. Mémoire de DEA en biologie végétale, Université Cheickh Anta DIOP, Dakar, Sénégal, p. 73.

FAO. 2017. Données de l'alimentation et de l'agriculture. www.fao.org. Consulté le 01/09/2019.

Gomgnimbou APK, Bandaogo AA, Coulibaly K, Sanon A, Ouattara S, Nacro HB. 2019. Effets à court terme de l'application des fientes de volaille sur le rendement du maïs (Zea mays L.) et les caractéristiques chimiques d'un sol ferralitique dans la zone sudsoudanienne du Burkina Faso. Int. J. Biol. Chem. Sci. 13(4): 2041-2052. DOI: https://dx.doi.org/10.4314/ijbcs.v13i4.11

Hema D. 2000. Analyse de la stabilité du rendement de variétés de maïs pour les zones sèches du Burkina Faso. Sécheresse, 11(3): 189-193.

Hiema SC. 2005. Caractérisation et classification de lignées de maïs (Zea mays L.). Mémoire de fin d'études, Université Polytechnique, Bobodioulasso, Burkina Faso, p. 103.

Hoopen MEP, Maïga A. 2012. Production et transformation du maïs. Collection ProAgro, 31p.

Kati Y. 2012. Contribution à la maîtrise de la fertilisation phosphatée des sols de montagne: cas des sols de vergers de pommiers d'Ichemoul (Aures-Algérie). Mémoire pour l'obtention du Diplôme de Magister en Sciences Agronomiques, Université El-Hadj Lakhdar-Batna, Institut des Sciences Vétérinaires et des Sciences Agronomiques. option: pédologie, p. 84.

Kiye Nkoy-Moke N, Lubini Ayingwe C, Luyindula Ndiku S, Likengelo Babelangi A. 2014. Amendement des sols, influence des fertilisants pour l'amélioration de la culture de Glycine max (L) Merril (soja). Int. J. Biol. Chem. Sci. 8(5): 2030-2041. DOI: http://dx.doi.org/10.4314/ijbcs.v8i5.9

Kocou MM. 2012. Effet du système sawah sur la production du riz et de la productivité de l'eau dans les bas-fonds de Bamé au Bénin. Mémoire de fin d'étude, Université de Parakou, Benin, p. 59.

Lafitte HR. 2002. Stress abiotique affectant le maïs. In : Le maïs en zones tropicales : amélioration et production. Collection FAO : Production végétale et protection des plantes; 97-108.

Mulaji KC. 2011. Utilisation des composts de bio-déchets ménagers pour l'amélioration de la fertilité des sols acides de la province de Kinshasa (République Démocratique du Congo). Thèse de Doctorat, Université de Liège Gembloux Agro-Bio Tech, p.220.

Muyayabantu GM, Kadiata BD, Nkongolo KK. 2012. Response of maize to different organic and inorganic fertilization regimes in monocrop and intercrop systems in a Sub-Saharan Africa Region. Journal of Soil Science and Environmental Management, 3(2): 42-48. DOI: $10.5897 /$ JSSEM11.079

N'da HA, Akanvou L, Kouakou CK. 2013. Gestion locale de la diversité variétale du maïs (Zea Mays L.) violet Tagouana au Centre-Nord de la Côte d'Ivoire. Int. J. Biol. Chem. Sci., 7(5): 2058-2068. DOI : http://dx.doi.org/10.4314/ijbcs.v7i5.23

Nyembo NKL, Useni SY, Chinawej KMD, Kaboza Y, Mpundu MM, Baboy LL. 2014. Amélioration des propriétés physiques et chimiques du sol sous l'apport combiné des biodéchets et des engrais minéraux et influence sur le comportement du maïs (Zea mays L. variété Unilu). J. Appl. Biosci., 74: 6121- 
6130.

DOI:

http://dx.doi.org/10.4314/jab.v74il.7

Ouédraogo J, Nacro HB, Ouédraogo E, Youl S, Sedogo M. 2014. Amélioration de la disponibilité du phosphore par la gestion de la macrofaune du sol : cas d'un lixisol en zone semi-aride du Burkina Faso. Int. J. Biol. Chem. Sci., 8(4): 1838-1846. DOI http://dx.doi.org/10.4314/ijbcs.v8i4.41

Siéné LAC, Coulibaly LF, Kouamé K, Condé M, Koné M, Aké S. 2018. Evaluation of the Effect of Three Types of Fertilizer on the Agronomic Potential of a Hybrid Variety of Maize (Zea mays L.), Grown in Korhogo Commune in Côte d'Ivoire. Journal of Experimental Agriculture International, $\quad \mathbf{2 8}(4)$ : $1-12$. DOI:10.9734/JEAI/2018/44727.

Soltner D. 2003. Les bases de la production végétale. Tome1. Le sol et son alimentation. Collection Sciences et techniques agricoles. 23ème édition; 472 p.

Tabetaoul M, Bessaoud R. 2009. La variabilité intra et inter-saisonnières des pluies, l'occurrence des phénomènes extrêmes (inondations, sécheresses et vagues de chaleur) et les séquences sèches limitant les rendements. In Adapter les agricultures au changement climatique, Mediterra (ed); 101-136.

Tine N. 2008. Caractérisation de la variabilité des rendements en maïs (Zea Mays L.) et leurs conditions d'élaboration dans la zone sud du département de Tambacounda. Diplôme d'études approfondies en biologie végétale, Université Cheickh Anta DIOP, Dakar, Sénégal, p.93.

Tshiabukole JPK. 2018. Evaluation de la sensibilité aux stress hydriques du maïs (Zea mays L.) cultivé dans la savane du Sud-Ouest de la RD Congo, cas de Mvuazi. Thèse de Doctorat, Faculté des Sciences Agronomiques, Département de Phytotechnie, Université Pédagogique Nationale, RD Congo, p. 162.

Yang J, Zhang J. 2006. Grain filling of cereals under soil drying. New Phytologist, 169: 223-236. DOI:10.1111/j.14698137.2005.01597.x

Yapi M. 2017. Agence national d'appui au développement rural. Fiche technicoéconomique du maïs, $5 \mathrm{p}$. 